\title{
Organization of controlled trials in paediatric liver disease
}

\author{
O. P. GRAY \\ M.B., M.Ch.B., F.R.C.P., D.C.H. \\ Department of Child Health, Welsh National School of Medicine, Heath Park, Cardiff
}

\begin{abstract}
Summary
There are very few controlled trials of treatment of liver disease in either adults or children. This is unfortunate because there are considerable uncertainties surrounding the effectiveness of the treatments in use. However, controlled trials present considerable difficulties to investigators in terms of organization and maintenance of enthusiasm. In order to gain the maximum information from trials it is imperative to categorize patients accurately. The planning of the trial requires an infinite attention to detail. The plan should be as simple as is possible to answer the questions. It is important that controlled trials should be restricted to 2 years, duration wherever possible. This allows the investigator's interest to be maintained. In planning trials, it is important to remember that when the trial is mounted, the recruitment of patients is often considerably less than that which was expected. Because of the inadequacy of knowledge of effectiveness of treatment in liver disease it appears to be unethical to do anything other than controlled trials, henceforth.
\end{abstract}

Krugman (1972) in summarizing the 1971 Seminar on Viral Hepatitis held at the International Children's Centre, presented the six aspects of viral hepatitis which had been discussed. They included the clinical aspects, pathology, epidemiology, aetiology, immunology and prevention of hepatitis. There was no section on treatment. This is hardly surprising as until recently the effective treatments of hepatitis and indeed liver disease generally, have been relatively few. Controlled trials of drugs used to treat liver diseases are unusual. Chalmers (1968) suggested that there are 50 uncontrolled trials to every controlled one. Discussing the treatment of hepatic coma he stated that the number of trials which had been controlled could be measured on the fingers of one hand. Yet there are outstanding challenges for study of treatment in liver disease in childhood, such as chronic hepatitis, hepatic coma, progressive neonatal hepatitis and Reyes Syndrome. The reasons for little work on treatment of these conditions lie not only on the paucity of effective therapies, but also from the serious difficulties presented to the investigator. These include both the relative rarity of the problems and the uncertain outcome. Dr Porter et al.'s (1972) report of the South-East England Study of Neonatal Hepatitis in 1971 concerned fifteen patients. Two years later Dr Mowat reported 100 cases which were seen in the King's College Hospital Unit from the same area. This is a testimony to the value of co-operative studies and investigations. The chronicity of some liver complaints poses serious obstacles to the evaluation of treatment and strains the fortitude of the investigator to the utmost. It is important that any investigation is conducted by physicians with considerable expertise in the disease under question. In the United Kingdom, there is a dearth of paediatricians with a special knowledge of liver disease. Unless a new treatment proves to be of immense benefit, controlled trials are mandatory. There are unfortunately various philosophical and practical impediments to using controlled trials which must be faced. The first is the physician's reluctance to accept that a new therapy which has been developed in the laboratory and confirmed by animal work could be more harmful than beneficial. Such a drug is usually regarded as an advance in treatment. It would appear instinctively to be more ethical to treat fresh cases with the new drug than randomize cases and compare with our present best treatment. Only a controlled trial will show whether the new drug is worse or better than the present best.

Controlled trials are often preceded by a pilot investigation which is undertaken to evaluate the snags and unseen complications. Naturally the results of a few consecutive cases are inspected and can prejudice the investigator to believe that the treatment is ineffective, or else so good that it would be unethical to deprive future patients of this new drug or treatment. Some of the early work on spina bifida can be criticized on this account. There is a very strong case to place the first patient of any treatment regime into a randomized controlled trial. Many have a secret aversion to undertaking controlled studies because of the fear that the numbers which may be required for definite conclusions to be reached could be excessive. It must be pointed out, however, that controlled trials do not always require randomization 
of large numbers of patients to gain a definite conclusion. If a new treatment presents a considerable advantage, this becomes rapidly clear. Penicillin, insulin and streptomycin did not require big trials. Psychological barriers to controlled trials are maintaining the amount of time required to set up a trial, the zeal which is necessary to continue its momentum and to recruit the great amount of cooperation which is required from other bodies. Sir Austin Bradford Hill is said to have remarked that it is impossible to undertake a successful clinical trial without paying a nauseating amount of attention to detail (Sutherland, 1972). An important pre-requisite to any drug trial is accurate diagnosis and categorization. Penicillin tried for all chest infections would require a big controlled trial before an advantage was apparent. If the trial was restricted to pneumococcal pneumonia, its advantage would be quickly apparent. The giving of quinine to reduce fever would require a very big trial to establish its effectiveness unless it happened to be conducted in malariarich country. In liver disease, where it is likely in the immediate future that treatment will be partially successful and the natural history variable, it is imperative carefully to select categories of patients and randomize within the clearly defined categories. The investigation of treatment in liver disease must involve the use of multicentric trials because of the relatively small numbers in one centre. These trials in themselves present severe organizational problems. However, there have been many successful multicentric trials in Great Britain often organized under the umbrella of the Medical Research Council. The present oncological trials and the former AngloAmerican Rheumatic Fever Trials are examples. Multicentric trials for some liver diseases are necessary also because some patients are too ill to move far to a big centre. For those too who have chronic liver disease, frequent long journeys will not readily be undertaken. One psychological disadvantage of the multicentric trial is that the imaginative and creative characteristics which are often found in the devoted investigator are restricted by the protocol of an agreed trial. A serious hazard is likely to arise when a trial is inadequate in any sphere, for this will soon be discovered and will quickly lead to friction amongst the investigators. On the other hand, if the trial is well designed and propagated it will generate enthusiasm and be widely applied.

It is highly important, therefore, that the planning of trials of treatment in liver disease is performed in the utmost detail. The initial phase of planning must concentrate on developing the hypotheses to be tested. Sometimes these are simple. Drug $X$ is superior to drug $Y$ in the present treatment of, for instance, chronic hepatitis. This is a very laudable hypothesis to test in a serious disease. More funda- mental hypotheses will prove more difficult to answer but will be infinitely more academically challenging, and ultimately more rewarding. These hypotheses should relate to aetiology initially and later treatment. After the hypotheses are established the appropriate questions are designed to test them. Here is the hard work. The reasons for the trial have to be made known. Then the methodology, organization and coordination is planned and later there is considerable exercise in liaising with the leaders in the various centres. Much work is required to arrive at standardization of observations and recording. It is important to choose people who are willing to subjugate their own individual views to that of the majority. Well designed planning for a trial may take 2 years. If there is any other way to answer the original question, avoid the trial!

It is important, when a multicentric trial is being operated, that the design is as simple as is feasible to answer the questions. Should the trial be established to refute or substantiate previous conclusions, it is mandatory that the protocols are strictly comparable. It is vital to decide on the end point of the trial at an early stage. With some conditions such as hepatic coma, this is relatively easy-death or not. However, with other conditions, such as neonatal hepatitis, the end point of an investigation is difficult to determine. It could be the return of abnormal liver function tests to normal.

In planning the trial it is wise to remember that even the most stalwart investigator has his interest taxed after a while. Frequently, it is impossible to maintain a general active interest after 2 years. Whenever possible a trial should so be designed that it can be completed within 2 years. However, this is not to rule longer trials out of court. The AngloAmerican Rheumatic Fever Trial organized by the Medical Research Council lasted many years. On the other hand, it has been suggested recently that diminished interest is likely to abort the big Australian Myocardial Infarction Trial (Hale, 1970). Already this trial has shown a trend in favour of a particular treatment (streptokinase) but in order to get a statistically significant difference, the trial will need to continue for another 7 years and have an extra 1400 patients. On the other hand, those working on acute liver problems can gain strength and confidence from the recent Boston trial on only fifty-three patients which showed improved survival from acute renal failure after treatment with intravenous essential $L$ amino acids and glucose compared with glucose alone (Abel et al., 1973).

Another difficulty with multicentric trials is that once a trial has begun, for various reasons, the number of patients eligible for the entry into it diminishes to about a tenth of that which was predicted before the trial. There may be doubts about the diagnosis. 
Patients can be excluded for various reasons. The consent may not be given by patient or the physicians concerned may feel unable to enter him into the trial. In the urokinase and urokinase/streptokinase pulmonary embolism trial (Sherry, 1973), ten patients were screened for every one entered into the trial. The Centre-Satellite concept has been developed in order to get the numbers available. Several hospitals were related to the Medical Centre for a cluster. This in its turn will relate to the Central Main Coordinating Committee. This enables an organization to develop successfully.

Dr Elwood, Medical Research Council Epidemiology Unit, Cardiff insists that if a research is to succeed it must be entertaining in the true sense of the word (Elwood, 1973). The investigators must take a delight and pride in it. He maintains that it is very useful to build in second generation investigations which satisfy a secondary interest of the peripheral investigator. However, if these second order investigations are to be included, they must on no account inflict further trauma on the patient nor in any way prejudice his health, otherwise the whole trial will be threatened. Examples of second generation questions could be that in any trial involving the use of steroids on young infants, what are the effects on head growth and intellectual function, the acquisition of circadian rhythms etc. This concept of providing entertaining research requires a search for the interest of the other investigators and accommodating these within the trials as far as is possible and reasonable. In many trials of course, the entertainment is provided by the possibility of a relatively rapid cure from the treatment itself.

In order to keep a trial going it is important to maintain good human relationships. This means establishing strong personal links with the various investigators, getting them up to a conference or a review meeting from time to time which should be as pleasant an occasion as possible. A newsletter to the people doing the day to day work is often appreciated. In order to deal with the diminishing interest of the central organizers it is useful to devolve much of the running of the trial to well trained clerical officers. These need not necessarily be medically trained people. Often those with training in industry or commerce are excellent in maintaining the smooth running of clinical trials.

Problems as to whether a particular controlled trial of a new treatment in liver disease is ethical or not centre around the probable benefit as against the risks expected. It is a matter of weighing possible gains against costs and these for patients generally and the individual patient before one in particular.
Sometimes the hazards of treatments are known, such as exchange transfusion or cortisone treatment. With some drugs the risks can at best be guessed. Azathioprine has known myelotoxic effects which are worse in patients with cirrhosis. What is not known is the long term effects of azathioprine. What are the risks of later malignant disease or, even more conjectural, what possible mutagenic effects are there on the gonads? It will take observation of at least one generation to determine this.

It was Lasagna (1969) who wrote 'The problem boils down to a sober weighing of costs and gains not preoccupation with moral cliches and stereotyped mottoes'. This viewpoint has been challenged as being 'the superficially soothing voice of moderation defying "medical progress" and casting it as the modern successor to the Golden Calf of the erring Israelites of old' (Thomson, 1972). Medical progress in the right sense of the term can only be achieved if all the time we place the individual patient in the forefront. The question that every doctor must ask himself is, 'What will most benefit my patient?'

I submit that whereas this is always the doctor's attitude, the very reason that a trial is being conducted means that the question is as yet unanswerable. The main ethical problem I see for trials of treatment in liver disease is whether it is ever justifiable not to do a controlled investigation using the best currently available treatment as the control.

\section{References}

Abel, R.M., BeCK, C.H., AвbotT, W.M., Ryan, J.A., BARNETT, G.O. \& FisCher, J.E. (1973) Acute renal failure: treatment with intravenous amino-acids and glucose. New England Journal of Medicine, 288, 695.

Chalmers, T.C. (1968) The management of hepatic comaa continuing problem. Medical Clinics North America, 52, 1475.

HALE, C.S. (1970) Streptokinase in acute myocardial infarction. Rationale and preliminary results of a combined therapeutic trial. Australian Annals of Medicine, 19, Suppl. 1, 63.

KRUGMAN, S. (1972) Summary of seminar on viral hepatitis. American Journal of Diseases of Children, 123, 435.

LASAGNA, L. (1969) Special subjects in human experimentation. Daedalus, 98, 449.

Porter, C.A., Haynes, D., Mowat, A.P. \& Williams, R. (1972) Etiologic factors in neonatal hepatitis. American Journal of Diseases of Children, 123, 300.

SherRY, S. (1973) Clinical trials with antithrombotic and thrombolytic agents. Principles and pitfalls. Thrombosis et Diathesis Haemorrhagica (Stuttg.), 29, 3.

Sutherland, I. (1972) In: The Therapeutic Choice in Paediatrics (Ed. by W. L. Burland and B. M. Laurence), p. 11. Churchill Livingstone, Edinburgh and London.

Thomson, W.A.R. (1972) In: The Therapeutic Choice in Paediatrics (Ed. by W. L. Burland and B. M. Laurence), p. 4. Churchill Livingstone, Edinburgh and London. 